\title{
Study of the Polarization Deterioration During Physics Stores in RHIC Polarized Proton Runs
}

\author{
Z. Duan and Q. Qin \\ Key Laboratory of Particle Acceleration Physics and Technology, \\ Institute of High Energy Physics, Chinese Academy of Sciences, \\ Beijing 100049, China \\ M. Bai \\ Forschungszentrum Juelich GmbH, 52425 Juelich, Germany \\ T. Roser \\ Brookhaven National Laboratory, Upton, New York 11973, USA \\ Published 29 February 2016
}

\begin{abstract}
As the only high energy polarized proton collider in the world, the Relativistic Heavy Ion Collider (RHIC) has achieved a great success in colliding polarized proton beams up to $255 \mathrm{GeV}$ per beam energy with over $50 \%$ average store polarizations for spin physics studies. With the help of Siberian snakes as well as outstanding beam control during the acceleration, polarization loss during acceleration up to $100 \mathrm{GeV}$ is negligible. However, about $10 \%$ polarization loss was observed between acceleration from $100 \mathrm{GeV}$ to $255 \mathrm{GeV}$. In addition, a mild polarization deterioration during long store for physics data taking was also observed. In this paper, studies in understanding the store depolarizing mechanism is reported, including the analysis of polarization deterioration data based on the past couple of RHIC polarized proton runs.
\end{abstract}

Keywords: RHIC; polarized proton beam; depolarization; physics stores.

PACS numbers: 29.20.D-, 29.27.Hj

\section{Introduction}

The Relativistic Heavy Ion Collider (RHIC) at the Brookhaven National Laboratory was designed to provide colliding beams of polarized protons up to a beam energy of $250 \mathrm{GeV}{ }^{1}$ Two types of polarimeters are employed in RHIC for complementary measurements of the beam polarization. The H-jet polarimeter ${ }^{2}$ is designed

This is an Open Access article published by World Scientific Publishing Company. It is distributed under the terms of the Creative Commons Attribution 3.0 (CC-BY) License. Further distribution of this work is permitted, provided the original work is properly cited. 


\section{Z. Duan et al.}

to operate continuously throughout a physics store, to give an absolute measurement of the average polarization with a statistic uncertainty of about $6-8 \%{ }^{3}$ The p-Carbon polarimeter, ${ }^{4}$ on the other hand, can take fast measurements of beam polarization for about two minutes and achieve a statistic uncertainty of about $4 \%$. Two p-Carbon polarimeters are currently in use for each RHIC ring, about four measurements of the beam polarization are taken for each physics store. The polarization variation during a physics store is characterized by the slope of the linear fit of these measurements versus time, which is called the polarization decay rate $\frac{d P}{d t} .^{3}$ The measurements of $\mathrm{p}$-Carbon polarimeters are calibrated with the absolute measurements by the $\mathrm{H}$-jet polarimeters. ${ }^{5}$

In past few polarized proton runs, colliding proton beams up to $255 \mathrm{GeV}$ with above $50 \%$ average store polarization have been supplied for physics data taking. 6 Negligible polarization loss has been achieved during acceleration to $100 \mathrm{GeV}$ with the help of dual snakes and precise beam controls. ${ }^{1,7}$ However, the polarization suffered a moderate loss due to the strong resonances between $100 \mathrm{GeV}$ and the store energy at $250 \mathrm{GeV}$ as well as $255 \mathrm{GeV} .^{8,9}$ In addition, a non-negligible polarization decay during a typical 8-hour physics store was also observed in polarized proton runs. The measured store polarization and the polarization decay rates are averaged over different fills in the same run conditions, as shown in Fig. 1. A moderate polarization deterioration is observed for both beams. It is also obvious that the polarization decay rate is much less for run13 than for run12 with the same machine lattice. Moreover, for run13 with the elens lattice, there is a large difference between the polarization decay rates for the two beams. In this paper, we present our analysis of the RHIC polarization data for past few runs, to understand the physical mechanisms of the observed polarization deterioration in RHIC physics stores.
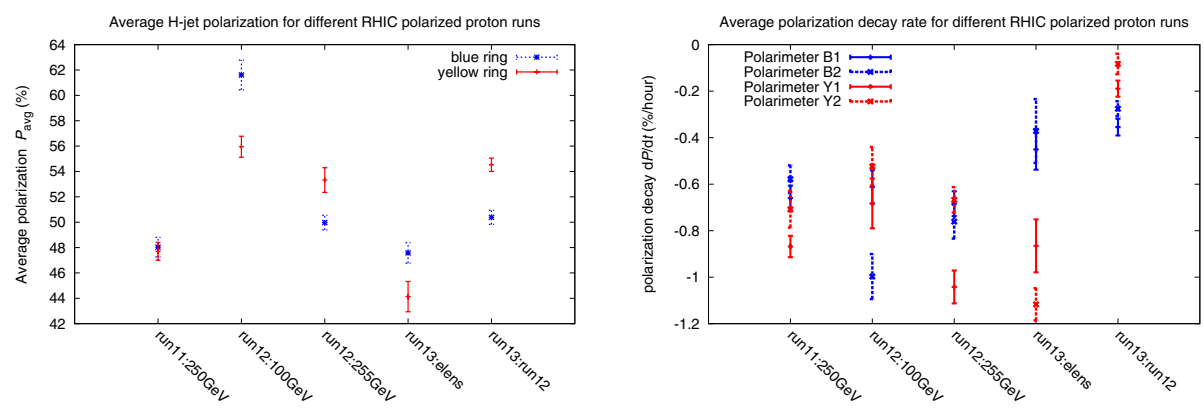

Fig. 1. The left plot shows the average polarization of different RHIC polarized proton runs measured by the $\mathrm{H}$-jet polarimeters, while the right plot shows the average polarization decay rates of different RHIC polarized proton runs measured by the four p-Carbon polarimeters, note that run13:elens corresponds to the first part of run13 with a baseline lattice optimized for elens operation, ${ }^{6}$ while run13:run12 corresponds to the later part of run13 when the same machne lattice of run12:255GeV was used. 


\section{Theoretical Formalism}

The polarization of a beam is in general defined as the ensemble avearge of all the spin vectors in the beam. Note that each polarization measurement takes over millions of revolutions in RHIC, so the time average of the beam polarization is more relevant for our concern. As an adiabatic invariant, ${ }^{10}$ the projection of a particle spin to the Invariant Spin Field (ISF) $\vec{n}(\vec{z}, \theta)^{11}$ is the contribution of this particle to the time averaged beam polarization. The long-term polarization is then defined as $^{12}$

$$
P_{\infty}=<<\vec{s} \cdot \vec{n}>\vec{n}>,
$$

where $\vec{s}$ is a particle spin vector, the first average is taken over the particles on the same initial phase space point, and the second one is averaging over the whole phase space. When the lattice tune is placed close to strong spin-orbit resonances, the "cone" of ISF distribution diverges away from $\vec{n}_{0}$, which leads to a reduction in the long-term polarization. As a result, the long-term polarization depends on the choice of the lattice tunes. The fractional parts of RHIC betatron tunes are set between the $2 / 3$ orbital resonance, and the $7 / 10$ snake resonance.

From the definition of the long-term polarization, we can see variation of either of the beam distribution in the phase space, or the ISF distribution can lead to the change of the long-term polarization. During acceleration, the leading effect is that the ISF distribution is changed due to the beam energy change. During physics stores with a fixed beam energy, however, machine tuning by the operators, power ripple, and other beam current dependent effects that modifies the lattice settings can change the ISF distribution. Moreover, the most relevant beam distribution variation to our concern is the emittance growth, which might also be important for the long-term polarization variation.

The most obvious current dependent effect is the beam-beam interaction, whose strength depends on the beam currents. It has three effects on the long-term polarization, the first is that beam-beam acts as an electromagnetic field and rotate the spin locally, the second is that beam-beam introduces a tune shift and an incoherent tune spread among the beam particles, the third is that beam-beam might cause the emittance growth in the long term. The first effect is negligible in general at RHIC, because the spin resonances are dominantly driven by the quadrupoles for such a high $G \gamma,{ }^{13}$ while the quadrupole component of the beam-beam kick is comparably weak in contrast with the normal quadrupoles in RHIC lattice. The second effect varies the tune distribution among the beam with the variation of the beam-beam parameter.

\section{Data Analysis}

The long-term polarization is a function of multiple variables, like the working point, beam emittances, spin tune on the closed orbit, beam-beam parameter, etc, but the form of the function is beyond our knowledge. Therefore, we attempted to 

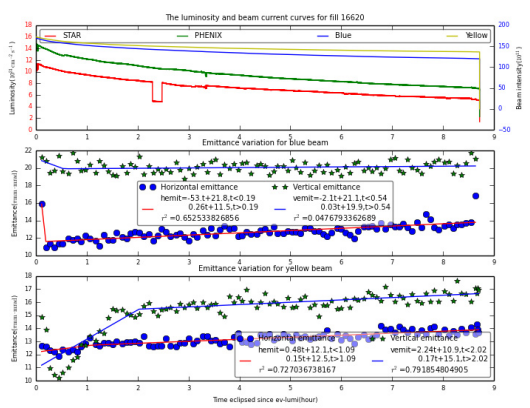

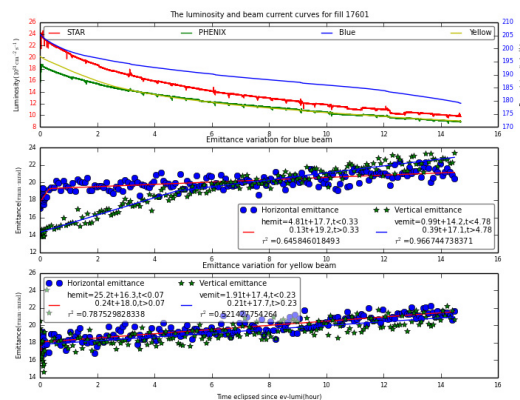

Fig. 2. The two plots shows the results in fill 17601 and 16620, respectively. And within each the upper plot shows the beam currents and luminosities variations, while the middle and lower plots show the blue and yellow emittances variations.

correlate the beam polarization data versus each of these parameters to have a better understanding of the leading effect behind the observed polarization deterioration in physics stores.

First, the correlation of the beam polarization and the behavoir of beam emittances was studied. Measured with four ionization beam profile monitors (IPM), the behavoir of the beam horizontal and vertical emittances vary among different fills. As shown in Fig. 2 are several typical plots of the variation of emittances together with the beam currents and luminosities, the emittance data were fitted by a piecewise linear curve, the initial emittance, the first and second slopes were then extracted and correlated against the beam average polarization, as well as the polarization decay rate. However, no obvious correlation was observed for these attempts.

Then the spin tune shift due to orbital imperfection was explored. In the ideal ring configuration with two diametrically opposed orthogonal snakes, the spin tune on the closed orbit is 0.5 . However, there are several effects ${ }^{14}$ that shift the spin tune away from 0.5. First, the imperfection in the snake current settings shifts the snake axis as well as the spin rotation angle, which can be optimized via the snake current scanning in the machine operation. Moreover, the horizontal orbital angle difference between the two snakes and the vertical orbital angle difference between the two spin rotators around one interaction point can also contribute to a shift in the spin tune. In run13, there was a $3 \mathrm{~mm}$ vertical orbit bump at IP6, which introduced a spin tune shift around 0.013. For run13, the closed orbit recorded by the BPMs were analyzed for each fill, and contribution to the spin tune shift from these orbital imperfections were computed and shown in Fig. 3. The maximal fillby-fill variation of these three contributions is around 0.005 . However, within the statistics of current RHIC polarization measurements, no clear correlation between polarization decay rate and spin tune shift was seen. 

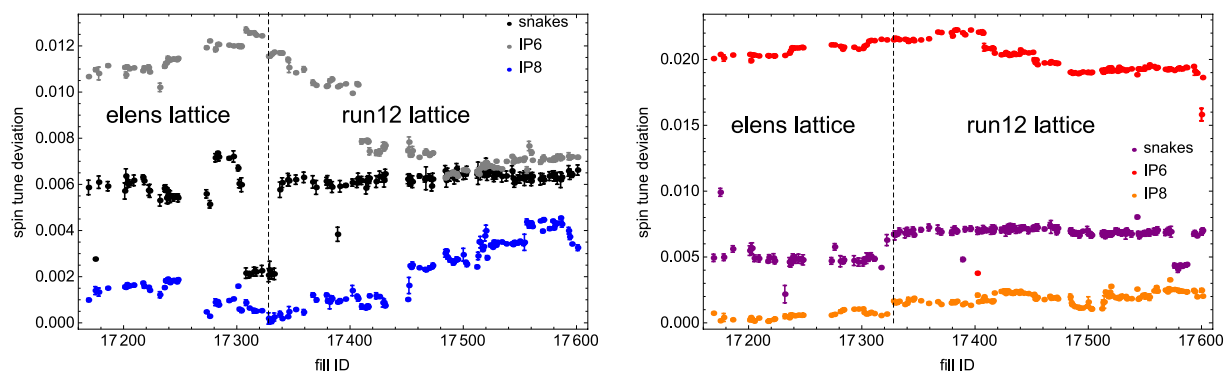

Fig. 3. Contributions to spin tune deviation from 0.5, from the orbital angle differences between snakes, spin rotators around IP6, and spin rotators around IP8 for run13 blue ring (left figure) and yellow ring (right figure). The two different run conditions with elens lattice setting, and the same lattice setting with run12, are indicated in the figure.

Next, we come to the analysis of betatron tunes. In physics stores, beam-beam interactions introduce an incoherent tune shift and spread among the beam particles, as well as coherent modes once the coherent motion is excited, which can be observed in the amplitude signal of the Beam Transfer Function(BTF) versus the scan frequency. Since currently the betatron tune of non-colliding bunches can not be directly measured in RHIC, the $\sigma$-mode measured via BTF is then the best approximation we can achieve, for the case with the same species and round beams. The location of $\sigma$-modes at the beginning of physics stores are extracted for all physics fills in run 12 and run 13 with beam energy $255 \mathrm{GeV}$, and a comparison of the fill-by-fill vertical $\sigma$-modes between run12 and run13 is shown in Fig. 4 . It was found that for $255 \mathrm{GeV}$ polarized proton runs, the working point setting at store in run 13 is in general lower than that of run12 with the same baseline lattice setting, which was due to the different tune feedback settings towards the end of rotator ramp when the two beams are cogged into collision. The larger vertical tune indicates
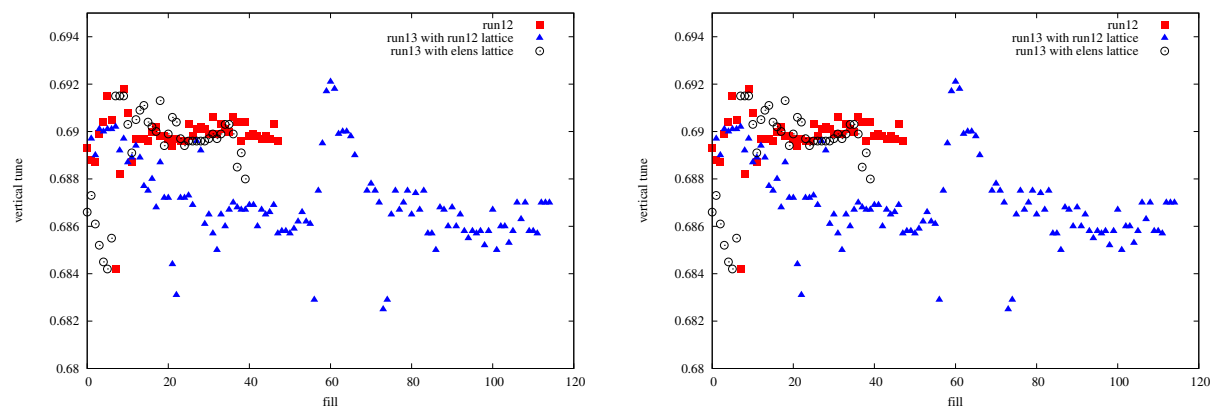

Fig. 4. The fill-by-fill vertical tunes approximated by the $\sigma$-modes for run12 and run 13 with the beam energy of $255 \mathrm{GeV}$, the left plot corresponds to the blue ring, and the right plot corresponds to the yellow ring. 
the closer to the strong $\nu_{s}=7 / 10$ snake resonance, which then could lead to larger polarization deterioration.

\section{Conclusion}

The physical mechanism behind the observed polarization deterioration in RHIC physics stores is explored in this work. The analysis of RHIC history data showed no clear correlation of the polarization deterioration versus the beam emittance behavior, or the spin tune shift due to orbital imperfection within statistics, while comparison between different run conditions indicate the polarization deterioration might be related to the working point settings. Simulation studies are underway to qualitively unveil the possible physical mechanisms, which will be reported elsewhere.

\section{Acknowledgments}

We highly appreciate Drs. D. Abell, D. P. Barber, E. Forest, Y. Luo, F. Meot, V. Ptitsyn, V. Ranjbar, D. Smirnov and many others for their instructive discussions. The author Z. Duan would like to express his gratitude for the hospitality he received during his stay in the Collider-Accelerator Department in BNL.

\section{References}

1. I. Alexseev et al., Design Manual - Polarized Proton Collider at RHIC (Brookhaven National Laboratory, 1998).

2. A. Zelenski et al., NIM A 536, 248 (2005).

3. D. Smirnov, Measurement of Proton Beam Polarization and Analyzing Power $A_{N}$ with Recoil Polarimeters at RHIC, in Proceedings of Spin 2012., 2012.

4. H. Huang et al., A p-Carbon CNI Polarimeter for RHIC, in Proceedings of PAC1999, 1999.

5. I. Nakagawa et al., Run05 Proton Beam Polarization Measurements by $p$ C-Polarimeter (ver. 1.1), RHIC/CAD Accelerator Physics Note 275, BNL (2008).

6. V. H. Ranjbar et. al., RHIC Polarized Proton Operation for 2013, in Proceedings of IPAC 2013, 2013.

7. Y. Derbenev and A. Kondratenko, Sov. Phys. Doklady 20, p. 562 (1976).

8. M. Bai el al., Phys. Rev. Lett. 96, p. 174801 (May 2006).

9. S. Y. Lee, Spin Dynamics and Snakes in Synchrotrons (World Scientific, Singapore, 1997).

10. G. H. Hoffstaetter, High Energy Polarized Proton Beams: A Modern View, no. 218 (Springer, 2006).

11. D. P. Barber, J. Ellison and K. Heinemann, Phys. Rev. ST Accel. Beams 7, p. 124002 (2004).

12. S. R. Mane, NIM A 528, 677 (2004).

13. S. R. Mane, Y. M. Shatunov and K. Yokoya, Reports on Progress in Physics 68, p. 1997 (2005).

14. V. Ptitsyn, M. Bai, T. Roser et al., Spin Tune Dependence On Closed Orbit in RHIC, in Proceedings of IPAC2010, 2010. 